\title{
Metabolic and Hematologic Effects and Immune Complex Formation Related to Pertussis Immunization ${ }^{1}$
}

\author{
CHRISANNA M. MINK, MATTI UHARI, DEAN A. BLUMBERG, MIKAEL KNIP, \\ KAREN LEWIS, PETER D. CHRISTENSON, MIEKO TOYODA, STANLEY C. JORDAN, \\ SEYMOUR R. LEVIN, AND JAMES D. CHERRY \\ Departments of Pediatrics and Medicine, UCLA School of Medicine, UCLA Medical Center, Cedars-Sinai \\ Medical Center, the Research and Medical Services, Veterans Administration Hospital, Los Angeles, California \\ 90024; and the Department of Pediatrics, University of Oulu, Oulu, Finland
}

\begin{abstract}
Selected metabolic, hematologic, and immunologic functions were evaluated in 3- to 6-mo-old Finish infants who received whole-cell pertussis-component diphtheria and tetanus toxoids and pertussis vaccine, adsorbed (DTP) vaccine, and in 4- to 6-y-old Los Angeles children who received either a licensed DTP vaccine or an acellular pertussis component DTP vaccine. One d after immunization, there was an increase in total leukocytes and neutrophils and a decrease in lymphocytes in all vaccinees. In 4- to 6-y-old children the leukocytosis and neutrophilia were greater in recipients who received the standard DTP vaccine than in vaccinees who received an acellular pertussis component DTP vaccine. In infants there was an increase in the mean plasma insulin concentration but no change in the glucose concentration $24 \mathrm{~h}$ after immunization; no increase in the mean plasma insulin was noted in the 4- to 6-y-old children. Three 4- to 6-y-old vaccinees had higher circulating immune complex concentrations after immunization and two of these children had high clinical reaction scores. The etiology of adverse reactions after DTP immunization is multifactorial. In contrast with findings in animals, our findings do not demonstrate a clinically significant effect due to lymphocytosispromoting factor on glucose metabolism in vaccinated children. Neutrophilia in vaccinees is probably due to endotoxin, and some reactions may be due to circulating immune complexes. (Pediatr Res 27: 353-357, 1990)
\end{abstract}

\section{Abbreviations}

AHG Eq, aggregated human gammaglobulin equivalent APDT, acellular pertussis component DTP vaccine CI, confidence interval

CIC, circulating immune complexes

DTP, diphtheria and tetanus toxoids and pertussis vaccine, adsorbed

FHA, filamentous hemagglutinin

LPF, lymphocytosis-promoting factor

LPS, lipopolysaccharide

WBC, white blood cell

In experimental animals, the administration of whole-cell pertussis vaccine causes hyperinsulinemia with hypoglycemia, leu-

Received July 20, 1989; accepted November 22, 1989

Correspondence and reprint requests: James D. Cherry, M.D., Department of Pediatrics, UCLA School of Medicine, Los Angeles, CA 90024

Supported by grants from Lederle Laboratories, Pearl River, New York, the Veterans Administration and the Emil Aaltonen Foundation, Tampare, Finland.

1 Previously reported in part in abstract form in Clin Res 37:191A, 1989. kocytosis with a predominant lymphocytosis, and marked adjuvancy in several immunologic systems (1-4). These effects are primarily due to the Bordetella pertussis antigen LPF but LPS (endotoxin) may also contribute to the metabolic, hematologic, and immunologic findings.

Many adverse clinical events occur after pertussis immunization in children, but the pathophysiology of these events is not well understood $(1,2,5)$. It has been suggested that some of these adverse events may be due to biologically active LPF and LPS that are present in routinely used DTP vaccines. There are, however, few data available that indicate that these antigens are in sufficient concentration in presently used DTP vaccines to produce hematologic or metabolic changes in vaccinated children.

In Japan, acellular pertussis vaccines have been used for the routine immunization of children since 1981 (6-8). These vaccines contain significantly reduced amounts of LPS and biologically active LPF, and their use has been associated with fewer vaccination side effects than occur in whole-cell pertussis vaccine recipients. Because of the paucity of information relating to hematologic and metabolic effects and immune complex formation associated with pertussis immunization in children, we have carried out two studies. In the Los Angeles area, as part of an evaluation of an APDT, we comparatively studied selected laboratory values in DTP and APDT immunized 4- to 6-y-old children. In Oulu, Finland, we carried out similar studies in routinely vaccinated 3 - to 6-mo-old infants.

\section{MATERIALS AND METHODS}

Subjects. In the Los Angeles area, the investigation was a substudy in a larger multicenter study in which the immunogenicity and reactogenicity of a standard whole-cell pertussis-component DTP vaccine was compared with a DTP vaccine that contained an acellular pertussis component (APDT) (9).

Thirty-three healthy 4- to 6-y-old children were enrolled. All had received four prior immunizing doses of DTP at the regularly scheduled intervals during the first 2 y of life. Informed consent was obtained from the parents after the design and purpose of the study were explained. This investigation was approved by the UCLA Human Subject Protection Committee. Sixteen children received APDT and 17 received DTP in a randomized double-blind fashion. One DTP recipient was excluded from further analyses because it was noted in retrospect that the child had gastroenteritis with onset before immunization.

The children were seen at $\mathrm{d} 0,1$, and 30 . At the initial visit (d 0 ), each child received $0.5 \mathrm{~mL}$ of vaccine administered into the deltoid muscle. The parents maintained a diary of local and 
systemic reactions for $10 \mathrm{~d}$ after immunization. A clinical reaction score was assigned in a manner as outlined previously (10). At each visit, blood samples were obtained to determine nonfasting plasma insulin, serum glucose, WBC and differential counts, CIC, and antibody concentrations. The time of the last meal before phlebotomy was recorded.

In Oulu, 40 subjects aged 3 to 6 mo (mean $3.4 \pm 0.8 \mathrm{mo}$ ) were recruited from children attending two city health centers for routine immunizations. DTP immunizations with a conventional whole-cell pertussis-component vaccine in Finland are scheduled at 3, 4, and 6 mo. Blood was obtained immediately before and on the day after immunization. Food intake data were recorded on a questionnaire before immunization and before the $\mathrm{d} 1$ blood sample was obtained. The study protocol was approved by the ethical committees of the University of Oulu and the Oulu Health Centers. Informed consent was obtained for study participation. Complete laboratory tests were not done for each subject visit due to inadequate blood specimens.

Laboratory techniques. In Los Angeles, serum glucose concentrations and WBC and differential counts were determined by standard methods at the UCLA Medical Center Clinical Laboratories. Insulin samples were collected in sodium fluoride anticoagulant tubes, and processed by RIA (11).

Circulating immune complexes were detected by Raji cell microenzyme immunoassay and $\mathrm{F}\left(\mathrm{ab}^{\prime}\right)_{2}$-anti-C3 microenzyme immunoassay using previously described methods (12-14). Both tests were used to increase diagnostic sensitivity because CIC formed in different children have different physiochemical characteristics and therefore may be demonstrable in one assay but not another (15). The antigenic composition of specific CIC was studied by their ability to be solubilized by preincubation with vaccine antigens. Solubilization causes a diminution in size and number of $\mathrm{CIC}$ with reduced binding in the assays. The tests were performed as previously described $(12,13)$ with the following modifications: the diphtheria toxoid was from lot PD-286, Department of Public Health, Division of Biological Laboratories, Boston; and BSA was included for control studies. Purified pertussis vaccine (lot $7 \mathrm{~b}$ ) was kindly provided by Dr. Charles Manclark of the Center for Biologics Evaluation and Research, Food and Drug Administration. Results are expressed as $\mu \mathrm{g}$ of $\mathrm{AHG} \mathrm{Eq} / \mathrm{mL}$.

In the 4- to 6-y-old children, antibodies to selected pertussis components of the vaccines were assayed by Lederle Biologicals (Pearl River, NY) using methods modified from Manclark et al. (16). Specifically, agglutinating antibodies were determined in microagglutination plates, and antibodies to LPF and FHA were determined by an indirect ELISA.

In Oulu, total WBC, neutrophil and lymphocyte counts, and blood glucose, growth hormone, and cortisol values were determined by the Clinical Laboratory, Oulu University Central Hospital. Plasma insulin and pancreatic glucagon concentrations were measured by RIA (17). Normal insulin values for infants after a 1- to $2-\mathrm{h}$ fast are below $25 \mu \mathrm{U} / \mathrm{mL}$. Inasmuch as the blood insulin concentration is affected by the concomitant blood glucose concentration and glucose values vary widely according to food intake, we analyzed changes in the insulin concentration independently of food intake by calculating the insulin/glucose ratio. Inasmuch as glucose causes no insulin secretion at concentrations less than $1.7 \mathrm{mmol} / \mathrm{L}$, the most useful ratio is insulin/ (glucose-1.7) (18).

Vaccines. The DTP vaccine used in Los Angeles was a single lot of licensed Lederle vaccine that met all current government requirements for this vaccine. The endotoxin concentration was 46875 endotoxin units $/ 0.5 \mathrm{~mL}$ as measured by the limulus amebocyte lysate assay (5 endotoxin units equal to $1 \mathrm{ng}$ of Escherichia coli endotoxin).

The APDT vaccine was formulated by Lederle Biologicals such that each dose contained 300 hemagglutinating units of Takeda acellular pertussis component. This component was manufactured by Takeda Chemical Industries, Osaka, Japan, and met all Japanese requirements for this vaccine. Components of the vaccine include LPF, FHA, and agglutinogens. This vaccine had an endotoxin content of 25 endotoxin units $/ 0.5 \mathrm{~mL}$.

The DTP vaccine used in Oulu was manufactured by the National Public Health Institute, Helsinki, Finland. The wholecell pertussis component is prepared and tested as suggested by the WHO (19). The vaccine contains 10 international opacity units $/ \mathrm{mL}$ and a minimum of 4 protective units $/ 0.5-\mathrm{mL}$ dose.

Statistics. Two categories of comparisons were made. 1) Group comparisons: differences in mean values for insulin, glucose, $\mathrm{CIC}$, and WBC and differential counts within vaccine groups were analyzed with the paired $t$ test, and similar differences between vaccine groups were studied using the two sample $t$ test. Nonparametric analogues, Wilcoxon tests, were used when appropriate. 2) Multivariate comparisons: comparisons of $\mathrm{CIC}$ and leukocytes between subjects with reactions and those without reactions were studied using Wilcoxon's rank sum test. Associations among CIC, antibody concentrations and WBC counts were measured with Spearman correlations. Bonferroni's method was used to adjust for multiple statistical tests with the same data in multivariate comparisons (20).

\section{RESULTS}

Leukocytes. In Los Angeles, 28 children (13 APDT and 15 DTP recipients) had values available for all 3 study d. A comparison of total WBC counts and absolute neutrophil and lymphocyte counts within a vaccine group over time (d 0,1 , and 30) and between vaccine groups (APDT versus DTP) at specific times is presented in Table 1 . In recipients of both APDT and DTP, there was a significant increase in mean absolute neutrophil counts from d 0 to 1 and a return to preimmunization values at d 30. In addition, DTP recipients had a significant increase in mean total WBC counts at $\mathrm{d} 1$ after immunization that returned to baseline at $\mathrm{d} 30$. Both groups had decreases in total lymphocyte counts from $\mathrm{d} 0$ to 1 , returning to preimmunization values at $d$ 30 ; this was significant in APDT vaccinees. The only significant difference between vaccine groups occurred on d 30: APDT recipients had a higher mean absolute lymphocyte count than DTP recipients.

No associations among total WBC, lymphocyte and neutrophil counts, and the presence of local of systemic reactions were noted. Similarly no relationship between WBC counts and LPF, FHA, or agglutinin antibody responses was noted.

The leukocyte findings in vaccinated infants in Oulu were similar to the findings in the older children in Los Angeles. D 0 and 1 total leukocyte counts were available for 32 subjects, and differential counts were performed for 31 subjects. The mean total leukocyte count increased from 11.1 to $12.9 \times 10^{3} / \mathrm{mm}^{3}$ after immunization $(p=0.001)$. This was due to a marked neutrophilia; the mean total neutrophil count increased from 2.5 to $5.5 \times 10^{3} / \mathrm{mm}^{3}$ after immunization $(p=0.001)$. The mean total lymphocyte count fell significantly after immunization from 7.7 to $6.5 \times 10^{3} / \mathrm{mm}^{3}(p=0.01)$.

Insulin and glucose. In Los Angeles, 12 APDT and 14 DTP recipients had values available for all 3 study d. A comparison of plasma insulin and serum glucose values within a vaccine group over time (d 0,1 , and 30$)$ and between vaccine groups (APDT versus DTP) at specific times is presented in Table 2. After immunization with both vaccines, there were no significant changes in mean plasma insulin or serum glucose concentrations at $\mathrm{d} 1$ or 30 when analyzed by the paired $t$ test. The marginally significant $(p=0.08)$ decrease in plasma insulin values in APDT recipients from $d 1$ to 30 reached significance $(p=0.04)$ with Wilcoxon's matched pairs signed rank test.

The timing of blood drawing after eating in the DTP and APDT recipients in Los Angeles were comparable. At $d 1$ and 30 only two instances of significantly elevated insulin values $(\geq 2$ $\mathrm{SD}$ above the 0 mean value) were noted; these both occurred 
Table 1. Comparison of total leukocyte counts and absolute neutrophil and lymphocyte counts in 4- to 6-y-old children within vaccine group over time (day 0, 1, and 30) and between vaccine groups [APDT $(n=13)$ versus DTP $(n=15)]$

\begin{tabular}{|c|c|c|c|c|c|c|}
\hline \multirow[b]{2}{*}{ Category } & \multirow{2}{*}{$\frac{\text { Day } 0}{\text { Mean }(95 \% \mathrm{Cl})}$} & \multirow{2}{*}{$\frac{\text { Day } 1}{\text { Mean }(95 \% \mathrm{CI})}$} & \multirow{2}{*}{$\frac{\text { Day } 30}{\text { Mean }(95 \% \mathrm{CI})}$} & \multicolumn{3}{|c|}{$\mathrm{p}$ values over time } \\
\hline & & & & $\begin{array}{c}\mathrm{D} \\
0 \text { versus } 1\end{array}$ & $\begin{array}{c}\mathrm{D} \\
0 \text { versus } 30\end{array}$ & $\begin{array}{c}\text { D } \\
1 \text { versus } 30\end{array}$ \\
\hline \multicolumn{7}{|c|}{ Total leukocytes $\times 10^{3} / \mathrm{mm}^{3}$} \\
\hline APDT & $\begin{array}{c}7.6 \\
(6.0-9.3)\end{array}$ & $\begin{array}{c}8.6 \\
(7.1-10.1)\end{array}$ & $\begin{array}{c}8.4 \\
(6.9-9.9)\end{array}$ & 0.16 & 0.30 & 0.85 \\
\hline DTP & $\begin{array}{c}7.2 \\
(6.3-8.1)\end{array}$ & $\begin{array}{c}9.4 \\
(7.8-11.1)\end{array}$ & $\begin{array}{c}7.2 \\
(5.8-8.5)\end{array}$ & 0.0005 & 0.96 & 0.02 \\
\hline$p$ & 0.56 & 0.41 & 0.21 & & & \\
\hline \multicolumn{7}{|c|}{ Total neutrophils $\times 10^{3} / \mathrm{mm}^{3}$} \\
\hline APDT & $\begin{array}{c}3.1 \\
(2.2-3.9)\end{array}$ & $\begin{array}{c}4.9 \\
(3.6-6.1)\end{array}$ & $\begin{array}{c}3.1 \\
(2.2-3.9)\end{array}$ & 0.0009 & 0.98 & 0.02 \\
\hline DTP & $\begin{array}{c}3.0 \\
(2.2-3.9)\end{array}$ & $\begin{array}{c}5.6 \\
(4.2-7.1)\end{array}$ & $\begin{array}{c}3.2 \\
(2.2-4.2)\end{array}$ & 0.0001 & 0.79 & 0.005 \\
\hline$p$ & 0.95 & 0.39 & 0.87 & & & \\
\hline \multicolumn{7}{|c|}{ Total lymphocytes $\times 10^{3} / \mathrm{mm}^{3}$} \\
\hline APDT & $\begin{array}{c}3.9 \\
(3.0-4.8)\end{array}$ & $\begin{array}{c}2.8 \\
(2.3-3.3)\end{array}$ & $\begin{array}{c}4.3 \\
(3.6-5.0)\end{array}$ & 0.008 & 0.35 & 0.0002 \\
\hline DTP & $\begin{array}{c}3.4 \\
(2.8-4.0)\end{array}$ & $\begin{array}{c}2.9 \\
(2.6-3.2)\end{array}$ & $\begin{array}{c}3.2 \\
(2.7-3.7)\end{array}$ & 0.09 & 0.62 & 0.07 \\
\hline$p$ & 0.31 & 0.70 & 0.01 & & & \\
\hline
\end{tabular}

Table 2. Comparison of plasma insulin and serum glucose values within vaccine group over time (day 0,1 , and 30$)$ and between vaccine groups [APDT $(n=12)$ versus DTP $(n=14)]$ in 4- to 6-y-old children

\begin{tabular}{|c|c|c|c|c|c|c|}
\hline \multirow[b]{2}{*}{ Category } & Day 0 & Day 1 & Day 30 & \multicolumn{3}{|c|}{$p$ values over time } \\
\hline & Mean $(95 \% \mathrm{CI})$ & Mean $(95 \% \mathrm{CI})$ & Mean $(95 \% \mathrm{CI})$ & $\begin{array}{c}\mathrm{D} \\
0 \text { versus } 1\end{array}$ & $\begin{array}{c}\mathrm{D} \\
0 \text { versus } 30\end{array}$ & $\begin{array}{c}\mathrm{D} \\
1 \text { versus } 30\end{array}$ \\
\hline \multicolumn{7}{|l|}{ Insulin $(\mu \mathrm{U} / \mathrm{mL})$} \\
\hline APDT & $\begin{array}{c}19.5 \\
(12.3-26.7)\end{array}$ & $\begin{array}{c}20.3 \\
(12.6-28.0)\end{array}$ & $\begin{array}{c}13.7 \\
(3.6-23.8)\end{array}$ & 0.83 & 0.32 & 0.08 \\
\hline DTP & $\begin{array}{c}15.1 \\
(7.1-23.2)\end{array}$ & $\begin{array}{c}13.2 \\
(9.0-17.3)\end{array}$ & $\begin{array}{c}10.1 \\
(5.3-14.9)\end{array}$ & 0.49 & 0.29 & 0.24 \\
\hline$p$ & 0.40 & 0.09 & 0.49 & & & \\
\hline \multicolumn{7}{|l|}{ Glucose $(\mathrm{mmol} / \mathrm{L})$} \\
\hline APDT & $\begin{array}{c}5.38 \\
(4.93-5.84)\end{array}$ & $\begin{array}{c}5.47 \\
(4.97-5.97)\end{array}$ & $\begin{array}{c}5.15 \\
(4.82-5.49)\end{array}$ & 0.64 & 0.27 & 0.26 \\
\hline DTP & $\begin{array}{c}5.63 \\
(4.87-6.38)\end{array}$ & $\begin{array}{c}5.63 \\
(4.94-6.33)\end{array}$ & $\begin{array}{c}5.18 \\
(4.83-5.53)\end{array}$ & 0.98 & 0.31 & 0.28 \\
\hline$p$ & 0.56 & 0.69 & 0.91 & & & \\
\hline
\end{tabular}

in one APDT recipient. The values were $49.1 \mu \mathrm{U} / \mathrm{mL}$ at $\mathrm{d} 1$ and $60.8 \mu \mathrm{U} / \mathrm{mL}$ at $\mathrm{d} 30$. This child had eaten $10 \mathrm{~min}$ before the $\mathrm{d}$ 1 blood drawing and 140 min before the $\mathrm{d} 30$ specimen collection. The child's glucose values at $d 1$ and 30 were 6.00 and 5.67 $\mathrm{mmol} / \mathrm{L}$, respectively.

In the DTP-vaccinated infants in Oulu, the glucose concentrations were similar before (mean $4.6 \mathrm{mmol} / \mathrm{L}$ ) and after vaccination $(4.7 \mathrm{mmol} / \mathrm{L})$. The mean insulin concentration, however, increased significantly from $9.9 \mu \mathrm{U} / \mathrm{mL}$ at $\mathrm{d} 0$ to $12.8 \mu \mathrm{U} / \mathrm{mL}$ at $\mathrm{d} 1$ after vaccination $(p=0.03)$. In addition, the mean ratio of insulin/(glucose-1.7) rose significantly in the infants from 3.4 at $\mathrm{d} 0$ to 4.3 at $\mathrm{d} 1$ after DTP vaccination $(p=0.03)$ indicating that the vaccination had a glucose-independent stimulation of insulin secretion. Two infants had insulin values of more than $25 \mu \mathrm{U} / \mathrm{mL} 1 \mathrm{~d}$ after vaccination $(25.5$ and $34.5 \mu \mathrm{U} / \mathrm{mL}$; the glucose values were 4.2 and $5.3 \mathrm{mmol} / \mathrm{L}$, respectively). No changes were noted in growth hormone, cortisol, or pancreatic glucagon values after immunization.

Circulating immune complexes. Circulating immune complexes ranged from 0 to $32360 \mu \mathrm{g} \mathrm{AHG} \mathrm{Eq/mL}$ in the $\mathrm{F}\left(\mathrm{ab}^{\prime}\right)_{2}$ anti-C3 assay and from 0.7 to $1764.4 \mu \mathrm{g} \mathrm{AHG} \mathrm{Eq/mL}$ in the
Raji cell assay. There was no marked median increase in CIC at d 1 or 30 in either assay after either APDT or DTP vaccine.

In the $\mathrm{F}\left(\mathrm{ab}^{\prime}\right)_{2}$-anti-C3 assay, there were three instances in which the pattern suggested a vaccine effect (Table 3). Two children had received DTP and one APDT. The APDT recipient had the highest clinical reaction score of all the APDT vaccinees, and one of the two DTP recipients had the highest clinical reaction score in this vaccine group. One subject (vaccinee 3 in. Table 3) had solubilization effects in the Raji cell assay suggesting that the CIC were due to diphtheria antigen-antibody complexes.

Table 3. Three vaccinees with pattern in $F\left(a b^{\prime}\right)_{2}$-anti-C3 assay that suggests vaccine effect

\begin{tabular}{|c|c|c|c|c|}
\hline \multirow[b]{2}{*}{ Vaccinee } & \multirow{2}{*}{$\begin{array}{l}\text { Type of } \\
\text { Vaccine }\end{array}$} & \multicolumn{3}{|c|}{$\begin{array}{c}\text { Circulating Immune } \\
\text { Complex Value }(\mu \mathrm{g} \text { AHG } \\
\mathrm{Eq} / \mathrm{mL})\end{array}$} \\
\hline & & D 0 & D 1 & D 30 \\
\hline 1 & APDT & 0 & 2026 & 0 \\
\hline 2 & DTP & 1772 & 4290 & 228 \\
\hline 3 & DTP & 0 & 2231 & 0 \\
\hline
\end{tabular}


In the CIC solubilization assays, all three vaccinees presented in Table 3 had increasing CIC with increasing concentrations of pertussis antigen.

Of note is the fact that one DTP recipient developed generalized urticaria. This child had low unchanging CIC in both assays.

No significant statistical associations were found between presence of reactions and $\mathrm{CIC}$ values in either the $\mathrm{F}\left(\mathrm{ab}^{\prime}\right)_{2}$-anti-C3 assay or the Raji cell assay at $\mathrm{d} 1$, or the change of CIC values in the same assays from $\mathrm{d} 0$ to 1 .

The results in the two CIC assays were ranked from low to high and the correlations with WBC counts were then examined. The only significant correlation was for elevated neutrophils and CICs in the Raji cell assay at d $30(p=0.05)$.

No significant correlations were found for comparisons at $\mathrm{d} 0$ and at 30 of CIC and mean levels of LPF and FHA antibodies.

\section{DISCUSSION}

The mechanisms by which pertussis vaccine causes common reactions are unknown. The varied effects of pertussis vaccine antigens in animals have led to causation theories for both established vaccine reactions and other temporally related adverse events $(1,2)$.

Recent studies in which whole cell pertussis antigen vaccines have been compared with Japanese DTP vaccines with acellular pertussis components have been useful in analyzing common reactions $(7,9,21-24)$. The acellular pertussis vaccines contain significantly less endotoxin than whole-cell vaccines and also less biologically active LPF (7). In the comparative trials, fever, local symptoms and common systemic symptoms were all significantly less frequent in the acellular vaccine recipients. None of these studies evaluated specific markers of endotoxin or LPF activity.

Endotoxin in humans produces leukocytosis with neutrophilia, and biologically active LPF is thought to cause leukocytosis with a lymphocytosis in patients with pertussis. In this study, recipients of both DTP and APDT had leukocytosis with neutrophilia suggesting an endotoxin effect without any evidence of an effect due to LPF. The response in the 4- to 6-y-old children was greater in DTP than APDT recipients but the difference between the two responses was small. This is surprising because the endotoxin content of the whole-cell vaccine was approximately 1800 times that of the acellular product as measured by the limulus amebocyte lysate assay.

The effect of LPF in animals on glucose metabolism has led to the consideration that hypoglycemia might occur in vaccinated children and that this could be the cause of irritability, persistent crying, hypotonic-hyporesponsive episodes, seizures, and perhaps other serious adverse events (1). There are few data at present that support the idea that hypoglycemia occurs in children in response to pertussis immunization. Furman et al. (25) have shown that plasma insulin concentrations were significantly higher in children with pertussis than in a group of controls; however, plasma glucose concentrations were normal in the two groups. Sen et al. (26) observed that DTP-vaccinated children had a blunted hyperglycemic response to epinephrine, and Hannik and Cohen (27) found that infants who received a concentrated pertussis vaccine (16 opacity units per dose) had significantly higher plasma insulin concentrations $8 \mathrm{~h}$ after vaccination than controls who received diphtheria and tetanus toxoid-polio immunization. Recently Sekura et al. (28) noted elevated insulin concentrations in four of 76 adult volunteers who received an experimental pertussis vaccine composed of purified LPF inactivated with hydrogen peroxide. The increased insulin values in two subjects were attributed to violation of the fasting protocol, and the other two elevations were not statistically different from the baseline values of the individual vaccinees.

In a clinical trial in adults, pertussis vaccine administration to insulin resistant diabetics resulted in a hypoglycemic effect (29). When LPF was administered i.v. to five healthy adults, no effect on blood glucose was noted; plasma insulin increased modestly, and this effect was significantly more pronounced in a glucose tolerance test after the administration of LPF than in a glucose tolerance test done before the administration of LPF (30).

In our study in 4- to 6-y-old children, no clinically relevant increase in plasma insulin or decrease in serum glucose was noted in either APDT or DTP recipients. It is possible that changes in both plasma insulin and serum glucose were missed because blood was obtained at only $\mathrm{d} 1$ and 30 and dietary intake was not rigidly controlled (although the dietary patterns of the children on $\mathrm{d} 0,1$, and 30 were remarkably similar). In the vaccinated Finnish infants a modest increase in plasma insulin was observed $1 \mathrm{~d}$ after immunization, similar to that noted by Hannik and Cohen (27) $8 \mathrm{~h}$ after immunization. However, no infants in our study or in the report by Hannik and Cohen (27) had concomitant hypoglycemia. It is possible that the insulin response to pertussis immunization occasionally results in varying degrees of hypoglycemia in infants. This could be a cause of persistent inconsolable screaming and the hypotonic-hyporesponsive state. However, in a study of six children with persistent crying and five children with hypotonic-hyporesponsive episodes we found no instances of hypoglycemia (31).

Recently members of our group noted a child with petechiae and urticaria after DTP immunization in whom tetanus and diphtheria antigen-specific CIC were noted (12). Because of this finding, we wondered if immune complexes could be found in association with other vaccine reactions or perhaps as part of the typical immune response. In our study only three of 25 vaccinees (12\%) had marked rises in immune complexes after immunization. The etiology of the immune complexes may have been diphtheria antigen administration in one subject. The increasing CIC noted with increasing additions of pertussis antigens in the solubilization assays were most likely due to high pertussis antibody concentrations. Two of these three children had high clinical reaction scores. Interestingly, one DTP vaccinee developed generalized urticaria and had low unchanging CIC in both assays.

The etiology of adverse reactions after DTP immunization is multifactorial. Some reactions are due to circulating immune complexes that may involve either toxoid or bacterial antigens (12). The degree of fever in vaccinees correlates directly with the endotoxin content of the vaccine administered (5). The finding of neutrophilia $1 \mathrm{~d}$ after immunization is probably a response to endotoxin in the vaccines, but the lack of a more marked difference between DTP and APDT vaccine recipients is surprising. Our findings do not demonstrate a clinically significant effect due to LPF on glucose metabolism in vaccinated children; this is in contrast to the findings in experimental animals (1). Modest insulin increases have been noted only in studies of infants (the Finnish infants in this report, and Ref. 27), perhaps indicating that a dose effect may be important.

Acknowledgments. The authors thank Dr. A. Holtzman and the physicians and staff of Descanso Pediatric Medical Group for assistance in study subject recruitment.

\section{REFERENCES}

1. Cherry JD, Brunell PA, Golden GS, Karzon DT 1988 Report of the task force on pertussis and pertussis immunization-1988. Pediatrics 81(suppl):939984

2. Furman BL, Sidey FM, Smith M 1988 Metabolic disturbances produced by pertussis toxin. In: Wardlaw AC, Parton R (eds) Pathogenesis and Immunity in Pertussis. J Wiley \& Sons, New York, pp 147-172

3. Munoz JJ 1988 Action of pertussigen (pertussis toxin) on the host immune system. In: Wardlaw AC, Parton R (eds) Pathogenesis a and Immunity in Pertussis. J Wiley \& Sons, New York, pp 173-192

4. Chaby R, Caroff M 1988 Lipopolysaccharides of Bordetella pertussis endotoxin. In: Wardlaw AC, Parton R (eds) Pathogenesis and Immunity in Pertussis. J Wiley \& Sons, New York, pp 247-271

5. Baraff LJ, Manclark CR, Cherry JD, Christenson PD, Marcy SM 1989 Analysis of adverse reactions to diphtheria and tetanus toxoids and pertussis vaccine by vaccine lot, endotoxin content, pertussis vaccine potency and percentage of mouse weight gain. Pediatr Infect Dis J 8:502-507 
6. Kimura M, Kumo-Sakai H 1988 Pertussis vaccines in Japan. Acta Paediatr Jpn 30:143-153

7. Sato Y, Kimura M, Fukumi H 1984 Development of a pertussis component vaccine in Japan. Lancet 1: 122-126

8. Noble GR, Bernier RH, Esber EC, Hardegree MC, Hinman AR, Klein D, Saah AJ 1987 Acellular and whole cell pertussis vaccines in Japan: report of a visit by US scientists. JAMA 257:1351-1356

9. Morgan CM, Blumberg DA, Cherry JD, Reisinger KS, Blatter MM, Blumer JL, Dekker CL, Stout MG, Christenson PD 1989 Comparison of acellular and whole-cell pertussis-component DTP vaccines: a multicenter doubleblind study in 4- to 6-year-old children. Am J Dis Child 144:41-45

10. Lewis K, Cherry JD, Sachs MH, Woo DB, Hamilton RC, Tarle JM, Overturf GD 1988 The effect of prophylactic acetaminophen administration on reactions to DTP vaccination. Am J Dis Child 142:62-65

11. Lundquist I, Fanska R, Grodsky G 1976 Interaction of calcium and glucose on glucagon secretion. Endocrinology 99:1304-1312

12. Lewis K, Jordan SC, Cherry JD, Sakai RS, Le CT 1986 Petechiae and urticaria after DTP vaccination: Detection of circulating immune complexes containing vaccine-specific antigens. J Pediatr 109:1009-1012

13. Jordan SC, Gautier E, Sakai R, Bahn L 1985 Quantitation of circulating immune complexes in human serum by the Raji cell and $F\left(a b^{\prime}\right)_{2}$-anti-C3 micro enzyme immunoassays. J Immunol Meth 83:363-370

14. Jordan SC, Sakai R, Malekzadeh MH, Pennisi AJ, Ettenger RB, Uttenbogaart $\mathrm{CH}$, Fine RN 1981 Circulating immune complexes in pediatric renal allograft rejection. Transplantation 31:190-194

15. Jordan SC 1985 Immune complexes in pediatric practice. In: Moss AJ (ed) Pediatrics Update. Elsevier, New York, pp 67-79

16. Manclark CR, Meade BD, Burstyn DG 1986 Serologic response to Bordetella pertussis. In: Rose NR, Friedman H, Fahey JL (eds) Manual of Clinical Laboratory Immunology. ASM, Washington, DC, pp 388-394

17. Knip M, Lautala P, Leppaluoto J, Akerblom HK Kouvalainen K 1983 Relation of enteroinsular hormones at birth to macrosomia and neonatal hypoglycemia in infants of diabetic mothers. J Pediatr 103:603-611

18. Turner RC, Oakley NW, Nabarro JDN 1971 Control of basal insulin secretion, with special reference to the diagnosis of insulinomas. Br Med J 2:132-135

19. WHO Expert Committee on Biological Standardization 1979 WHO Tech Rep Ser 638:60-80

20. Miller RG 1981 Simultaneous Statistical Inference, 2nd ed. Springer-Verlag, New York, pp 67-70
21. Lewis K, Cherry JD, Holroyd HJ, Baker LR, Dudenhoeffer FE, Robinson RG 1986 A double-blind study comparing an acellular pertussis component vaccine with a whole cell pertussis component DTP vaccine in 18 month old children. Am J Dis Child 140:872-876

22. Anderson EL, Belshe RB, Bartram J, Gurwith M, Hung P, Leuner M, Vernon SK 1987 Clinical and serologic responses to acellular pertussis vaccine in infants and young children. Am $\mathcal{J}$ Dis Child 141:949-953

23. Edwards KM, Lawrence E, Wright PF 1987 Diphtheria, tetanus and pertussis vaccine. A comparison of the immune response and adverse reactions to conventional and acellular pertussis components. Am J Dis Child 140:867871

24. Pichichero ME, Badgett JT, Rodgers Jr GC, McLinn S, Trevino-Scatterday B, Nelson JD 1987 Acellular pertussis vaccine: immunogenicity and safety of an acellular pertussis vs. a whole cell pertussis vaccine combined with diphtheria and tetanus toxoids as a booster in 18- to 24-month old children. Pediatr Infect Dis J 6:352-363

25. Furman BL, Walker E, Sidey FM, Wardlaw AC 1988 Slight hyperinsulinaemia but no hypoglycemia in pertussis patients. J Med Microbiol 25:183-186

26. Sen DK, Arora S, Gupta S, Sanyal RK 1974 Studies of adrenergic mechanisms in relation to histamine sensitivity in children immunized with Bordetella pertussis vaccine. J Allergy Clin Immunol 54:25-31

27. Hannik CA, Cohen H 1979 Changes in plasma insulin concentration and temperature of infants after pertussis vaccination. In: Manclark CR, Hill JC (eds) International Symposium on Pertussis. US DHEW, NIH, Bethesda, MD, pp 297-299

28. Sekura RD, Zhang Y-I, Roberson R, Acton B, Trollfors B, Tolson N, Shiloach J, Bryla D, Muir-Nash J, Koeller D, Schneerson R, Robbins JB 1988 Clinical, metabolic, and antibody responses of adult volunteers to an investigational vaccine composed of pertussis toxin inactivated by hydrogen peroxide. $J$ Pediatr 113:806-813

29. Dhar HL, Dhirwani MK, Sheth UK 1975 Pertussis vaccine in diabetics requiring high dosage insulin. Br J Clin Pract 29:119-120

30. Toyota T, Kai Y, Kakizaki M, Sakai A, Goto Y, Yajima M, Ui M 1980 Effects of islet-activating protein (IAP) on blood glucose and plasma insulin in healthy volunteers (phase 1 studies). Tohoku J Exp Med 130:105-116

31. Blumberg DA, Morgan CM, Read KL, Leach CT, Holtzman A, Levin SR, Baraff LJ, Cherry JD 1989 A preliminary report of an ongoing surveillance study of persistent crying (PC) and hypotonic-hyporesponsive episodes (HHE) following routine DTP immunization. Clin Res 37:190A(abstr) 